\title{
PHIL implementation of a decentralized online OPF for active distribution grids
}

This paper was downloaded from TechRxiv (https://www.techrxiv.org).

\section{LICENSE}

CC BY-NC-SA 4.0

SUBMISSION DATE / POSTED DATE

$23-11-2021 / 29-11-2021$

\section{CITATION}

Cornejo, Martin; Mohapatra, Anurag; Candas, Soner; Peric, Vedran S. (2021): PHIL implementation of a decentralized online OPF for active distribution grids. TechRxiv. Preprint.

https://doi.org/10.36227/techrxiv.17065193.v1

$\mathrm{DOI}$

10.36227/techrxiv.17065193.v1 


\title{
PHIL implementation of a decentralized online OPF for active distribution grids
}

\author{
Martín Cornejo, Anurag Mohapatra, Soner Candaş, Vedran S. Perić \\ Technical University of Munich \\ Munich, Germany \\ \{martin.cornejo, anurag.mohapatra, soner.candas, vedran.peric\}@tum.de
}

\begin{abstract}
This paper demonstrates a Power Hardware-in-theLoop (PHIL) implementation of a decentralized optimal power flow (D-OPF) algorithm embedded into the operations of two microgrids connected by a tie line. To integrate the static behavior of the optimization model, a two layer control architecture is introduced. Underneath the dispatch commands from the D-OPF, a primary control scheme provides instantaneous reaction to the load dynamics. This setup is tested in the PHIL environment of the CoSES Lab in TU Munich. In the experiment, the two microgrids cooperatively optimize their operation through an ADMM based unbalanced D-OPF. The operations is then benchmarked against the exclusive use of primary control, without D-OPF. The decentralized approach outperforms, but also shows minor inefficiencies of integrating optimization methods into the realtime operation of the system.
\end{abstract}

Index Terms-Optimal power flow, power hardware-in-theloop, distribution grids, decentralized control, real time control

\section{INTRODUCTION}

Smart grid and microgrid technologies have emerged as a response to the disruptive integration of distributed energy resources (DER) in power distribution systems. The individual control and optimal coordination of the fast dispatchable inverters connected to the DER makes microgrid operation a challenge. Traditionally in a transmission grid, the optimal power flow (OPF) has been a tool of choice for the operator to dispatch the generators to minimize losses and achieve the best economic performance within the grid constraints. In a distribution grid context, online or real-time OPF can allow fast redispatch of controllable power electronics generation at the household level, in order to leverage local electricity market mechanisms.

However, smart distribution grids also bring their associated concerns with scalability, robustness and privacy, which lends emphasis towards a decentralized implementation scheme [1]. Unlike conventional methods, in which a central controller collects all the data and performs the computations, decentralized algorithms are carried out in multiple local controllers by exchanging limited information to their proximal peers. This allows for an online decentralized OPF (D-OPF) scheme for

The construction of the CoSES laboratory was supported by Deutsche Forschungsgemeinschaft (DFG) through the project "Flexible reconfigurable microgrid laboratory" under Project number 350746631. The work of Vedran Perić was supported by Deutsche Forschungsgemeinschaft (DFG) through the project "Optimal Operation of Integrated Low-Temperature Birdirectional Heat and Electric Grids (IntElHeat)" under Project number 450821044. prosumers who do not want to share information with other neighboring microgrids and yet want to take advantage of an optimal operation of their generation.

While this topic has been extensively studied, to the best knowledge of the authors, a Power Hardware-in-the-Loop (PHIL) implementation in a realistic grid setting has not yet been attempted. Porting the online D-OPF algorithm into a real time (RT) PHIL application requires further considerations, generally neglected in the simulation models. Another challenge is the integration of the static optimization problem into a RT control scheme for PHIL testbeds. This implementation scheme must be practical and, most importantly, should not depart from reality, to further the claim that online D-OPF schemes can be used in real microgrids.

In this paper we present a PHIL implementation scheme and experimental validation of an online D-OPF method within the Center for Combined Smart Energy Systems (CoSES) lab. CoSES at TU Munich was established to research smart multienergy systems in an emulated environment [2]. The laboratory emulates a distribution grid through a PHIL system, [3] and therefore lends a valid platform to testbench the online D-OPF implementation in a realistic environment.

The rest of this paper is structured as following. In Section II we introduce the proposed methodology for the validation. The decentralized OPF algorithm is presented, alongside some considerations from the authors to integrate D-OPF in a PHIL environment. Section III gives an insight on the implementation on the CoSES-lab. Section IV] presents the experiment, results and discussion. Finally, Section $\mathrm{V}$ closes with the conclusions and the outlook for further research.

\section{Methodology}

\section{A. Decentralized OPF algorithm}

Many decentralized methods have been implemented for OPF applications [1]. Arguably, the state-of-the-art in decentralized OPF algorithms constitutes the ADMM-OPF and has been therefore extensively adopted in previous works [4]-[8].

The Alternating Direction Method of Multipliers (ADMM) performs distributed optimization by decomposing an optimization problem into smaller sub-problems and iteratively solving these while sharing information on a few common variables [9]. The advantage over other distributed algorithms is, that consensus-ADMM enables a fully decentralized OPF 
implementation with no need of a central controller to orchestrate the optimization [10].

ADMM can be integrated into the OPF by partitioning the power grid, by means of a network decomposition or by splitting it in administrative zones (the independently managed micro-grids and distribution networks). These local power grids can be also referred to as clusters. Through the decomposition, each bus of the network is allocated to a specific cluster and to locally replicate the cluster-coupling power lines, each local grid takes a copy of the directly neighboring buses.

Over the set of all grid partitions $\mathcal{M}$, each local grid $\{m, n, \ldots\} \in \mathcal{M}$ can be individually optimized and the solution will ultimately correspond to the full-grid optimization as long as the voltages of all peripheral buses match among all clusters. ADMM exploits this relation for the distributed optimization of the OPF. First, the common global variables $\bar{v}_{i}$ are introduced, representing the voltages of all shared buses on cluster periphery. For the global optimum to be preserved, ideally, the peripheral voltages of all cluster $m \in \mathcal{M}$ should be consistent with the global variables:

$$
\bar{v}_{i}-v_{i}^{(m)}=0, \quad \forall i \in \mathcal{N}_{\varphi}^{(m)}
$$

The goal is to iteratively optimize the local OPF sub-problems, exchange the resulting peripheral voltages, update the global variables and repeat until all clusters agree on these within a margin of tolerance, hence having solved the global OPF problem.

For the local optimization, the voltage consensus (1) is introduced as a soft constraint in the form of an augmented Lagrangian. The objective function of a local OPF is extended from the active power generation cost $f\left(\mathbf{p}_{\mathbf{g}}\right)$ to the following:

$$
\begin{aligned}
\mathcal{L}_{\rho}\left(\mathbf{p}_{\mathbf{g}}, \mathbf{v}_{\varphi}, \overline{\mathbf{v}}_{\varphi}, \boldsymbol{\lambda}\right)=f\left(\mathbf{p}_{\mathbf{g}}\right) & +\boldsymbol{\lambda}^{T}\left(\overline{\mathbf{v}}_{\varphi}-\mathbf{v}_{\varphi}\right) \\
& +\frac{\rho}{2}\left\|\overline{\mathbf{v}}_{\varphi}-\mathbf{v}_{\varphi}\right\|_{2}^{2},
\end{aligned}
$$

where $\rho>0, \boldsymbol{\lambda}$ is the local Lagrangian multipliers, $\mathbf{p}_{\mathbf{g}}$ is the active power generation, $\overline{\mathbf{v}}_{\varphi}$ and $\mathbf{v}_{\varphi}$ are the global and local variables of the cluster peripheral bus voltages. Following the consensus ADMM method, for each iteration $k$, each local-OPF is solved with step-fixed global variables $\overline{\mathbf{v}}_{\varphi}^{(k)}$ and multipliers $\boldsymbol{\lambda}^{(k)}$.

$$
\begin{array}{ll}
\min _{\mathbf{p}}, \mathbf{q}_{\mathbf{g}}, \mathbf{v} & \mathcal{L}_{\rho}\left(\mathbf{p}_{\mathbf{g}}, \mathbf{v}_{\varphi}, \overline{\mathbf{v}}_{\varphi}^{(k)}, \boldsymbol{\lambda}^{(k)}\right) \\
\text { s.t. } & \\
\sum_{g \in \mathcal{G}_{i}} s_{g}-\sum_{d \in \mathcal{D}_{i}} s_{d}=v_{i} \sum_{k \in \mathcal{N}} Y_{i k}^{*} v_{k}^{*}, & \forall i \in \mathcal{N}_{\chi} \\
v_{i}^{l} \leq\left|v_{i}\right| \leq v_{i}^{u}, & \forall i \in \mathcal{N} \\
p_{g}^{l} \leq p_{g} \leq p_{g}^{u}, & \forall g \in \mathcal{G} \\
q_{g}^{l} \leq q_{g} \leq q_{g}^{u}, & \forall g \in \mathcal{G} \\
\mathbf{v}_{0}=\hat{\mathbf{v}}_{0} &
\end{array}
$$

The new local peripheral voltages $\mathbf{v}_{\varphi}^{(k+1)}$, from the local OPF iteration, are collected across all local grids to update the values of the global variables, by taking the average over all local peripheral voltages. A fully decentralized method can be achieved by exchanging the peripheral voltages only between adjacent clusters and locally performing the averaging step.

Multipliers are then updated locally based on the globallocal voltage mismatch scaled with the penalty parameter:

$$
\boldsymbol{\lambda}^{(k+1)}=\boldsymbol{\lambda}^{(k)}+\rho\left(\overline{\mathbf{v}}_{\varphi}^{(k+1)}-\mathbf{v}_{\varphi}^{(k+1)}\right)
$$

To measure the mismatch error among clusters and between iterations, two indicators are introduced - the primal residual $r$, which highlights the error between global and local variables, and the dual residual $s$, which underlines the deviation of the local variables from the previous to the next iteration.

$$
\begin{aligned}
r^{k} & =\left\|\overline{\mathbf{v}}_{\varphi}^{k+1}-\mathbf{v}_{\varphi}^{k+1}\right\|_{2} \\
s^{k} & =\rho\left\|\overline{\mathbf{v}}_{\varphi}^{k+1}-\overline{\mathbf{v}}_{\varphi}^{k}\right\|_{2}
\end{aligned}
$$

The algorithm stops when both residuals are below a desired tolerance $0<\epsilon \ll 1$ in all clusters $m \in \mathcal{M}$.

A disadvantage of ADMM is that the convergence speed is highly dependent on the penalty parameter $\rho$, and a bad choice could critically slow down the convergence. To counter this, an adaptive penalty is introduced via the residual balancing strategy, as proposed in [9] and extended in [11].

\section{B. Unbalanced $O P F$ formulation}

ADMM-OPF can be implemented agnostic from the OPF formulation, leaving it on the judgement of the implementer, to find the most fitting formulation for the use-case. Under this context, it is important to take into account that distribution grids have more demanding modeling requirements compared to transmission networks, as these should be able to accurately replicate an unbalanced state and the system losses.

The SDP-OPF formulation is chosen as it has proven its effectivity and has been extensively adopted for decentralized OPF applications, especially in the context of unbalanced power distribution systems [1], [6]-[8]. SDP-OPF has the advantage that, thanks to its convexified formulation, for 3phased radial distribution networks no modeling detail is sacrificed while the global optimum is searched [12], [13]. This also makes it possible to assess the feasibility and the quality of the solutions.

This paper follows the chordal reduced SDP-OPF formulation [14], which takes advantage of a technique that leverages sparsity and simplifies the computational complexity, significantly reducing the convergence time.

\section{PHIL considerations for online D-OPF}

Integrating D-OPF into a power system operation requires an interface capable of merging the optimization process with the RT control. The challenge lies in the different time domains for the two processes. The optimization models live in a static world, and it takes some time for the algorithm to compute the results, while the control has to perform the commands in real-time.

The PHIL experiment design must therefore be cognizant of few key considerations for a practical and safe implementation of the online D-OPF scheme. 
Local Stability of PHIL layer: The PHIL layer must have a local primary control strategy or a slack connection to the grid, to maintain a generation equal to the demand. This is necessary to mitigate against the load changes as the generators wait for the new optimization setpoints to arrive. It is also necessary to handle the mismatch in generation and load due to measurement errors and subsequent small deviations in the active power calculation.

Asynchronous operation of layers: The PHIL and optimization layers have to run asynchronously using common communication interfaces. This means that the two layers cannot stop or throw an error waiting for the other layer to respond. On the PHIL side, the setpoints from the D-OPF layer should combine with the primary control to give the setpoint to the generator. Thematically this concept is similar to a traditional primary and secondary control for generators. The primary control reacts instantaneously to local load changes and a slower power change command, from any form of secondary control (in this case the D-OPF), redispatches the generators.

Flexibility in system configuration: Multiple experiments would be required to thoroughly investigate the efficacy and consequences of using the online D-OPF to provide generation setpoints. Therefore the PHIL, optimization and interface design should not be rigidly programmed to suit one specific parameter set or one specific grid setup. Furthermore, the interface layer should be ideally flexible to be combined with any other setpoint generation tool, instead of the ADMM based online D-OPF, to increase re-usability of the work in a laboratory.

These points are revisited in the Section III where the specifics of the RT implementation of online D-OPF in CoSES lab is discussed.

\section{IMPLEMENTATION IN COSES}

\section{A. PHIL setup}

Detailed description of the electrical and control setup of CoSES can be found in [2] and [3]. A brief description, relevant to the scope of this paper, is provided in this section. The lab setup emulates a Low voltage (LV) distribution grid of up to seven electrical prosumer households. The grid consists of ten LV buses with cable segments connecting them, which can be rearranged based on the topology needs. The location of the prosumers with reference to the LV buses can be shifted easily in a patch cabinet. Two tap changing transformers connect the lab grid to the Munich public supply grid.

The prosumer behaviour is emulated by seven Egston COMPISO units (CSU) which are bi-directional four leg inverters supplied by a separate power feedback circuit [15]. Each Egston CSU is connected to a prosumer bus and receives setpoints from the CoSES control system through a SFP link. The separation of the CoSES LV grid and feedback grid keeps the power consumption of the lab to only the ohmic losses even while operating at rated power.

The control infrastructure of CoSES comprises of National Instruments (NI) embedded hardware, the RT deployment environment VeriStand (VS) [16] and its associated APIs. Six NI PXI controllers are spread across the lab as distributed RT agents. One controller, denoted henceforth as grid controller, collects the LV grid bus voltage and prosumer currents. Two further RT controllers send setpoints to the seven Egston CSUs over a SFP link card. These two controllers can be split as the two clusters used in the D-OPF algorithm and are known as Egston controllers in this paper.

The VS environment hosts the different RT targets and the associated field IOs. The grid controller runs at $10 \mathrm{kHz}$ RT execution rate, while the Egston controllers operate at $5 \mathrm{kHz}$. PXI targets cannot inherently exchange information between each other as this cannot be implemented as a RT task. Therefore an asynchronous ring called, Reflective Memory Network (RMN) is used to exchange information between CoSES controllers at a maximum jitter of $1 \mathrm{kHz}$. VS allows compiled models from $\mathrm{C} / \mathrm{C}++$, MATLAB, Simulink or LabVIEW and exposes their IOs to the grid measurements and Egston CSU setpoints. The VS Engine maintains the RT execution rate of the models, measurements and setpoints. Time synchronisation is provided between the six RT targets and the seven Egston CSU, which allows for accurate power injection in grid connected mode.

\section{B. D-OPF implementation}

The OPF and ADMM-OPF models is implemented in Julia and JuMP, using the interior-point conic solver MOSEK, to solve the SDP-OPF. For the ADMM implementation, the two local OPF models for the clusters shown in Fig. 1 are set up in two Windows PCs running independent Julia instances. The message exchange between the two ADMM clusters in Julia during the algorithm execution is achieved through TCP-socket connections and data serialization.

The optimization activity of the operation is enclosed in a static feedback loop. The clusters update the loads in the model from grid measurements, then jointly perform the ADMM-OPF and sent the optimal power setpoints to their corresponding Egston controllers. To interact with the RT targets, for both measurements and setpoints, the Julia instances rely on a Julia-LabView-VS bridge enabled through the VS API. The Julia instances communicate with the VS API using TCP connections in a JSON format.

Within the VS environment, a Simulink-compiled model for power measurement and PLL is implemented on the grid controller. In the Egston controllers, Simulink-compiled models are used for each CoSES prosumer in the cluster. These models convert power setpoints to precise current injection waveforms which are sent to the respective Egston CSU. The designated generators within the Egston CSUs, are provided with two setpoints from primary and secondary control respectively. A local cluster control is implemented to reflect the change in the local load from the base load conditions on the primary control power setpoint. If there are more than one generators in a cluster, they equally share the change in the load. The D-OPF output is taken as a secondary control setpoint, as mentioned in Sec. III. Importantly, when D-OPF is activated and a new setpoint is sent to the cluster, the primary control sets the 


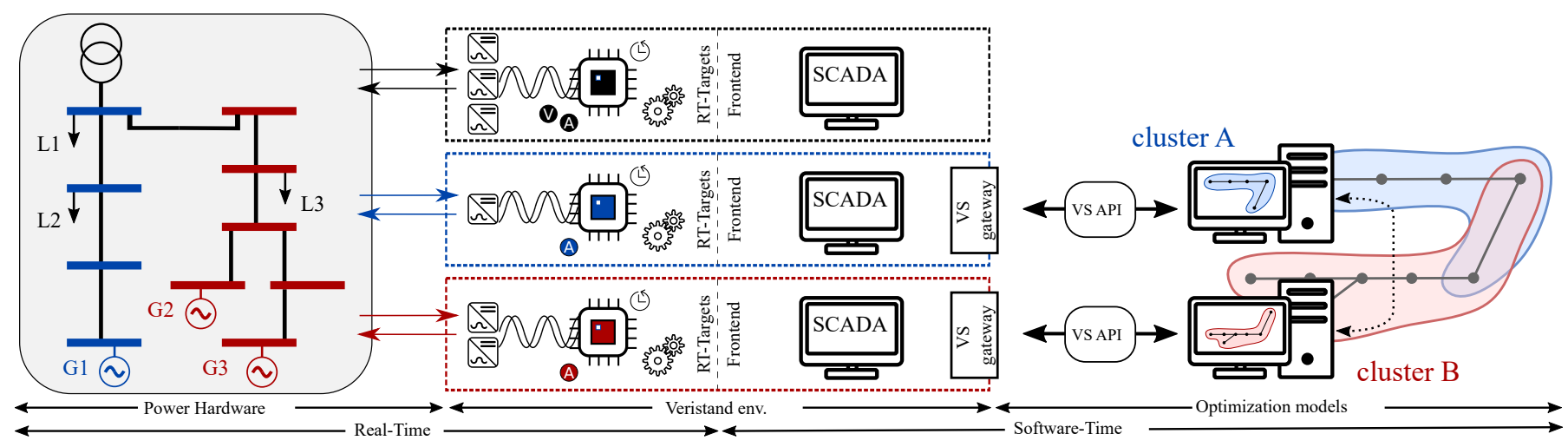

Fig. 1. D-OPF implementation in CoSES: Optimization (left), RT-Control (center), (left)

base load to the current measured load in the cluster. The primary control then dynamically changes the generation to match the load changes with respect to the new base load, until the next D-OPF setpoint arrives. Thus, a combination of the primary and secondary control setpoint, keeps the generation and load balance, irrespective of the rate of load changes or optimization delays.

\section{EXPERIMENT AND RESULTS}

\section{A. Experiment design}

The experiment takes a look into a distribution feeder divided into two clusters, both of which are organized as individual microgrids. Each microgrid has its own loads and generators, as well as an own control and monitoring system. It is assumed that the microgrid operators are interested in minimizing the cost of operating their system. However, the customers in the clusters do not necessarily want to share own information with another operator for a global optimization to work. Such motivations are common in current data privacy landscape and thus online D-OPF can be implemented here as a solution. The grid topology is represented in the leftmost graphic in Fig. 1, and the technical details are summarized in Table [

Two experiment runs are conducted for this paper to illustrate the PHIL validation of D-OPF. In the first scenario, the optimization is turned off and the two clusters are required to be self-sufficient with their generation needs. The primary control mechanism described in Section [II] is used for this scenario. The real life analogue can be a microgrid EMS to monitor the loads and adjust the generation setpoints based on a participation factor $\kappa_{g}$ and base power $p_{g}^{r e f}$.

In the second scenario, we activate the online D-OPF layer of secondary control in addition to the primary control

TABLE I

GENERATOR DATA

\begin{tabular}{|c|c|c|c|c|c|}
\hline Gen & Cluster & $\begin{array}{c}\mathbf{P}_{\max } \\
{[\mathbf{k W}]}\end{array}$ & $\begin{array}{c}\mathbf{Q}_{\max } \\
{[\mathbf{k V A R}]}\end{array}$ & $\begin{array}{c}\text { Cost } \\
{[\$ \mathbf{k W h}]}\end{array}$ & $\kappa_{\mathbf{g}}$ \\
\hline Grid & $\mathrm{A}$ & - & - & $10.0 p$ & - \\
G1 & $\mathrm{A}$ & 5.0 & \pm 4.0 & $6.0 p+0.4 p^{2}$ & 1.0 \\
G2 & $\mathrm{B}$ & 5.0 & \pm 4.0 & $4.0 p+0.4 p^{2}$ & 0.715 \\
G3 & $\mathrm{B}$ & 5.0 & \pm 4.0 & $3.0 p+0.5 p^{2}$ & 0.285 \\
\hline
\end{tabular}

for the cluster, for the same load profile. The experiment begins with zero secondary control setpoint and the primary control setpoint set according to the base load conditions. The D-OPF layer takes a snapshot of the loads and returns to the ADMM-based optimization algorithm. Once finished, the optimization returns a secondary control setpoint for the generators. The primary control's base load is now reset to the current measured load. Any subsequent changes to the load from that moment until the new D-OPF setpoints arrive, is handled by the primary control based on the participation factor of the generator.

The loads are emulated through Egston CSUs and are assembled from the HTW Berlin representative household load profiles [17]. These profiles consist of three-phase active and reactive power timeseries with 1 minute resolution. Each load is the aggregation of 10 individual households of the dataset, with the data time segment for the experiment taken from February 1, starting at 19:00. Both scenarios are run for 30 mins. The generation and load profiles, change in generation in between scenarios and power exchanged between clusters are plotted in the next section.

\section{B. Results}

Primary control only: The generators follow the load profile shape, with cluster A covering the internal loads from its only generator and cluster B sharing the local demand between the two generators according to their $\kappa_{g}$, as shown in Fig. 2. As expected, almost no power exchange, $P_{t i e}$ between clusters or with the grid is observed and is seen in Fig. 3. The operation is stable but as a consequence of the over-proportional contribution of the generator $\mathrm{G} 1$, in covering the total grid demand, the operational expenses grow unnecessarily excessive. The total cost at the end of the experiment run is $\$ 0.78$, from which cluster A contributes about $71 \%$.

With D-OPF: Just as with the previous run, the generation roughly mirrors the load course and is shown in fig. 2 This time however, with a much more balanced distribution of the generator participation, a lower total operational cost of $\$ 0.73$ (a reduction of about 6\%) is achieved. In Fig. 3, we see that the generation from $\mathrm{G} 1$ is reduced while the generation from G2 and G3 is increased, when the D-OPF is activated. The exchange between clusters is now prominent in Fig. 3 . 

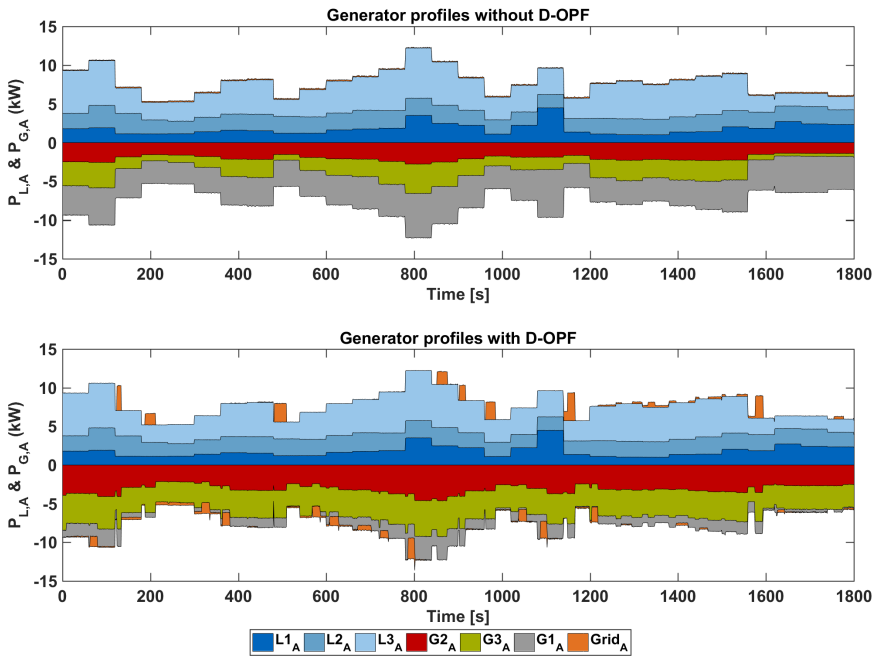

Fig. 2. Comparison of generation profiles with and without OPF

while the grid exchange is also noticeable in Fig. 2. The $P_{\text {tie }}$ power flows mostly from cluster B to A, curtailing the need to produce more from generator $\mathrm{G} 1$.

The grid involvement on the other hand is a side effect from the D-OPF wrapping implementation. When the load changes significantly while the optimization is in progress, by the time the results arrive these no longer correspond with the actual state and difference has to be compensated from the grid feeder. This mismatch is corrected a few seconds later with the arrival of new optimization results. In our experiment the grid import signifies a great cost penalty. Even though the overall occurrence is short, the cost contribution adds to $\approx 15 \%$ of total cost. The performance of the optimization algorithm is therefore critical to the reliability of the operations. For the case of our implementation, one instance of ADMMOPF takes in average $18.5 \mathrm{~s}$ and $\approx 205$ iterations to solve, including optimization time and communication overhead. This is significantly more than the central approach SDP-OPF, which takes instead a fraction of a second.

\section{CONCLUSION}

We have implemented a D-OPF scheme in a PHIL environment, taking some consideration relevant for a real deployment of the algorithm. The proposed control architecture operates in two layers, the upper level computes the optimal generator setpoints through the D-OPF and sends them to the lower layer, which ensures the system stability through real-time primary control. The interface between both layers should be redundant so that the layers don't depend on each other for their operation. Allowing this interface to be flexible permits to easily interchange optimization models and control algorithms.

The operation scheme is validated through a set of PHIL experiments in the CoSES microgrid-lab. The D-OPF scenario outperformed the individualistic approach consisting of exclusively the primary control. However, there are certain inefficiencies due to changing load as the optimization is getting computed. This serves as an open question to solve
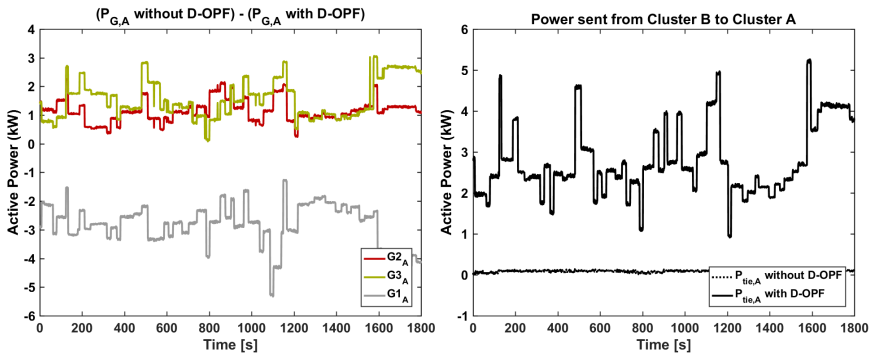

Fig. 3. Change in generator power (left) and tie line power (right) with and without D-OPF

in the future work on the topic for a seamless integration of online D-OPF in CoSES microgrid lab.

\section{REFERENCES}

[1] D. K. Molzahn, F. Dorfler, H. Sandberg, S. H. Low, S. Chakrabarti, R. Baldick, and J. Lavaei, "A Survey of Distributed Optimization and Control Algorithms for Electric Power Systems," IEEE Transactions on Smart Grid, vol. 8, pp. 2941-2962, Nov. 2017.

[2] V. S. Perić, T. Hamacher, A. Mohapatra, F. Christiange, D. Zinsmeister, P. Tzscheutschler, and C. Aigner, "CoSES Laboratory for Combined Energy Systems At TU Munich," IEEE Power and Energy Society General Meeting, 2020.

[3] A. Mohapatra, V. S. Peric, and T. Hamacher, "PHIL Infrastructure in CoSES Microgrid Lab," submitted to PES GM 2022, http://dx.doi.org/ 10.36227/techrxiv. 17065184

[4] T. Erseghe, "Distributed Optimal Power Flow Using ADMM," IEEE Transactions on Power Systems, vol. 29, pp. 2370-2380, Sept. 2014.

[5] M. Kraning, E. Chu, J. Lavaei, and S. Boyd, "Dynamic Network Energy Management via Proximal Message Passing," Foundations and Trends $®$ in Optimization, vol. 1, no. 2, pp. 73-126, 2014.

[6] E. Dall'Anese, H. Zhu, and G. B. Giannakis, "Distributed Optimal Power Flow for Smart Microgrids," IEEE Transactions on Smart Grid, vol. 4, pp. $1464-1475$, Sept. 2013.

[7] E. Dall'Anese, S. V. Dhople, B. B. Johnson, and G. B. Giannakis, "Decentralized Optimal Dispatch of Photovoltaic Inverters in Residential Distribution Systems," IEEE Transactions on Energy Conversion, vol. 29, pp. 957 - 967, Dec. 2014.

[8] Q. Peng and S. H. Low, "Distributed algorithm for optimal power flow on an unbalanced radial network," in 2015 54th IEEE Conference on Decision and Control (CDC), (Osaka), pp. 6915-6920, IEEE, Dec. 2015.

[9] S. Boyd, "Distributed Optimization and Statistical Learning via the Alternating Direction Method of Multipliers," Foundations and Trends in Machine Learning, vol. 3, no. 1, pp. 1-122, 2010.

[10] S. Candas, K. Zhang, and T. Hamacher, "A Comparative Study of Benders Decomposition and ADMM for Decentralized Optimal Power Flow," in 2020 IEEE Power \& Energy Society Innovative Smart Grid Technologies Conference (ISGT), (Washington, DC, USA), pp. 1-5, IEEE, Feb. 2020

[11] B. Wohlberg, "ADMM Penalty Parameter Selection by Residual Balancing," arXiv:1704.06209 [cs, eess, math], Apr. 2017. arXiv: 1704.06209.

[12] S. H. Low, "Convex Relaxation of Optimal Power Flow-Part I: Formulations and Equivalence," IEEE Transactions on Control of Network Systems, vol. 1, pp. 15-27, Mar. 2014.

[13] S. H. Low, "Convex Relaxation of Optimal Power Flow-Part II: Exactness," IEEE Transactions on Control of Network Systems, vol. 1, pp. 177-189, June 2014.

[14] L. Gan and S. H. Low, "Convex relaxations and linear approximation for optimal power flow in multiphase radial networks," in 2014 Power Systems Computation Conference, (Wrocław, Poland), pp. 1-9, IEEE, Aug. 2014.

[15] Egston Power, "COMPISO Power Hardware in the Loop," Nov. 8, 2021 [Online].

[16] National Instruments, "NI Veristand", 2018.

[17] T. Tjaden, J. Bergner, J. Weniger, and V. Quaschning, "Repräsentative elektrische Lastprofile für Wohngebäude in Deutschland auf 1-sekündiger Datenbasis." https://pvspeicher.htw-berlin.de/ veroeffentlichungen/daten/lastprofile/ 